\title{
Putting the "M" in Breastfeeding Medicine
}

\author{
Alison Stuebe
}

W ORLDWIDE, $>90 \%$ of women initiate breastfeeding, and more than half continue breastfeeding for at least 12 months after birth. Caring for the breastfeeding dyad thus requires providers to understand how breastfeeding affects, and is affected by, essentially any health condition that can affect women of childbearing age.

Advancing this understanding is a central part of the mission of The Academy of Breastfeeding Medicine's mission-and to reach that goal, we have work to do. Most breastfeeding research focuses on milk composition and the effects of optimal feeding on infant growth and development; mothers are conspicuously absent. Indeed, public health goals target the proportion of infants that "are exclusively breastfed," leaving the mother who breastfeeds out of the equation. Following a movement in other disciplines, including Maternal-Fetal Medicine and Maternal and Child Health, it is time to put the "M" back into Breastfeeding Medicine.

Mothers do the work of breastfeeding, and that work is substantial. Exclusive milk production requires $600-700 \mathrm{kcal}$ a day, ${ }^{1}$ and exclusively breastfeeding mothers spend 8.5 hours more per week than formula-feeding mothers feeding and caring for their infants. ${ }^{2}$ To reconcile these demands with women's underlying health conditions, we need evidencebased strategies that consider holistic needs.

And yet, review articles and medical society guidelines on chronic health conditions and reproduction typically focus on pregnancy and offer little guidance on lactation. It is expected that authors will discuss cardiometabolic, endocrine, and immunological adaptations of pregnancy and how these alter, or are altered by, comorbid diseases. For lactation, however, in the vast majority of cases, the discussion begins and ends with infant medication exposure through breast milk. This information is certainly necessary, but it is far from sufficient, to appropriately care for the breastfeeding mother-baby dyad.

What might holistic guidance look like? Here are six key principles to consider:

(1) Risk of congenital anomalies, fetal growth restriction, and preterm delivery. Both maternal chronic diseases and medications are associated with an increased risk of birth defects as well as pregnancy complications, fetal growth restriction, and preterm birth. Establishing breastfeeding in the context of a congenital anomaly or medically complex birth may require support for the mother to express milk, or assistance to accommodate at-breast feeding, for a medically fragile infant.

(2) Transition to extrauterine life and initiation of lactation. Maternal disease can impact neonatal physi- ology through placental transfer of medications, glucose, antibodies, and other bioactive molecules, affecting the newborn's transition to extrauterine life. Maternal health conditions can also disrupt the endocrinology of breast development and of milk synthesis, resulting in delay of lactogenesis II as well as reduced or absent milk production.

(3) Maternal medications, milk synthesis, and milk composition. Emerging studies have characterized the "omics" of human milk composition and explored variations in milk macronutrients, oligosaccharides, hormones, and microbiome. These differences may or may not be adaptive, and we should take care not to interpret basic science studies to presume that "different" milk is "inferior" milk. Maternal medications can also affect on blood flow to the breast and influence hormonal signals that regulate milk production.

(4) Physiological impact of breastfeeding and maternal health. The endocrine and immunological changes of lactation may aggravate or alleviate maternal diseases. The endocrine effects of exclusive breastfeeding, including elevated prolactin and reduced estrogen and progesterone, are distinct from mixed feeding, and may have different effects on maternal disease course. Nutrient demands also impact maternal health, particularly for women with malabsorptive disorders.

(5) Practical demands of breastfeeding. Exclusive breastfeeding is demanding, and this investment of time may be difficult to sustain for women with chronic health conditions. Night-time feedings may be especially challenging for women for whom sleep is a medical need. These medically necessary periods of rest can result in breast overfilling and engorgement, and strategies are needed to support draining the breasts with minimal disruption of maternal sleep. For some women, getting sufficient sleep to prevent complications such as mania, postpartum psychosis, or recurrent seizures ${ }^{3}$ may impact milk production. Other health conditions may affect the mechanics of feeding - a woman with rheumatoid arthritis, for example, may benefit from occupational therapy assistance to position her baby at breast.

(6) Lactation duration, intensity, and long-term maternal health. In observational studies, greater duration and intensity of breastfeeding is associated with substantial differences in women's health across the lifespan. These population-level differences likely reflect both 
the effects of breastfeeding on maternal physiology, and the extent to which being able to sustain breastfeeding is a marker for maternal health and wellbeing.

There are some promising examples of holistic clinical guidance: Edenborough et al. ${ }^{4}$ address the effects of maternal cystic fibrosis on milk composition, maternal nutritional needs, bone loss during lactation, and the importance of hands-on support to ensure that the mother continues her cystic fibrosis treatment while adjusting to life with a newborn. In an article on women with epilepsy, Voinescu and Pennell ${ }^{3}$ discuss the importance of maternal sleep, as well as considerations for safe infant positioning during feeding in women at risk of seizure. And in a recent article in Annals of Surgical Oncology, ABM members Helen Johnson and Katrina Mitchell review multidisciplinary management of lactation in breast cancer survivors and women with a new diagnosis. ${ }^{5}$

To enable breastfeeding, physicians and other health care providers need to consider the whole mother-baby dyad. That includes addressing hands-on material support for the mother and infant in the "4th Trimester" after birth. Let us work together to put the " $M$ " into Breastfeeding Medicine, both by advancing clinical research and by engaging each woman and her family to craft a sustainable strategy that centers their values and preferences, alongside the importance of human milk and the well-being of mother and infant.

\section{References}

1. Butte NF, King JC. Energy requirements during pregnancy and lactation. Public Health Nutr 2005;8:1010-1027.

2. Smith JP, Forrester R. Maternal time use and nurturing: Analysis of the association between breastfeeding practice and time spent interacting with baby. Breastfeed Med 2017; 12:269-278.

3. Voinescu PE, Pennell PB. Delivery of a personalized treatment approach to women with epilepsy. Semin Neurol 2017; 37:611-623.

4. Edenborough FP, Borgo G, Knoop C, et al. Guidelines for the management of pregnancy in women with cystic fibrosis. J Cyst Fibros 2008;7 Suppl 1:S2-S32.

5. Johnson HM, Mitchell KB. Breastfeeding and breast cancer: Managing lactation in survivors and women with a new diagnosis. Ann Surg Oncol 2019;26:3032-3039.

-Alison Stuebe, MD, MSc

President, Academy of Breastfeeding Medicine

Alison Stuebe, MD, MSc, is president of the Academy of Breastfeeding Medicine. She is professor of Maternal and Fetal Medicine at the University of North Carolina School of Medicine and Distinguished Professor of Infant and Young Child Feeding at the Gilling's School of Global Public Health. The President's Corner reflects the opinions of the author, not the Academy of Breastfeeding Medicine as a whole. 